\title{
Personal space and distance misperception: Implications of a novel observation
}

\author{
DAN ZAKAY \\ Tel Aviv University, Tel Aviv, Israel \\ LESLIE A. HAYDUK \\ University of Alberta, Edmonton, Alberta, Canada \\ and \\ YEHOSHUA TSAL \\ Tel Aviv University, Tel Aviv, Israel
}

\begin{abstract}
Subjects tried to halt an approaching or departing experimenter at a prespecified distance. In both the approaching and departing conditions, the subjects halted the mobile person at distances exceeding the preselected target distance, but the distance misestimation was greater for the approaching condition. This observation is inconsistent with current theories of personal space. This may be because most personal-space studies emphasize the inner boundary of personal space by investigating minimal acceptable distances or intrusions into personal space, whereas the phenomenon described here occurs at the outer boundary of personal space. Distance misestimation may lead to an alternative method of measuring personal space and emphasizes the necessity of investigating the phenomenological and subjective aspects of spatial behavior.
\end{abstract}

Personal space has been defined as the area individuals maintain around themselves into which others cannot intrude without arousing discomfort (Hayduk, 1978); it is often measured as the distance people choose to keep between themselves and others (Russell \& Ward, 1982). The distances people maintain are influenced by factors such as room size, furniture arrangements, the relative status of the participants, and the interactional histories of the participants (Hayduk, 1983). People whose spaces have been invaded usually attempt to flee or withdraw physically from the situation, but they may also withdraw psychologically or perceptually. Terry and Lower (1979), for example, have shown that the more subjects' spaces are invaded, the less they gaze at the intruder.

McNamara, Ratcliff, and McKoon (1984) and Stevens and Coupe (1978) have shown that the accuracy of geographical-distance estimation is influenced by motivational factors. This led us to wonder whether distance misestimation could be used to measure perceptual withdrawal resulting from personal-space preferences. We wanted to determine whether distance perception was distorted (underestimated) more in an invading condition than in a departing condition.

The authors wish to thank O. Lider, D. Binet, and N. Arnon for their assistance with data collection. Leslie A. Hayduk is in the Department of Sociology at the University of Alberta. Correspondence should be addressed to Dan Zakay, Department of Psychology, Tel Aviv University, Ramat Aviv, Tel Aviv 69978, Israel.

\section{METHOD}

Thirty-two female and 32 male undergraduate students at Tel Aviv University volunteered to participate in the experiment. (Two females and 1 male declined our invitation.) The subjects ranged from 20 to 30 years of age, and all had normal or corrected-to-normal vision. Four males and 4 females were assigned at random to each of the eight experimental conditions created by crossing the subject's sex with the target/experimenter's sex and with an approaching versus departing condition. The subjects and experimenters were strangers, and the entire experiment was conducted in an open outdoor plaza to avoid the influences of furniture and room structure.

The subjects were told that they were participating in an experiment to determine people's ability to measure the distance to a human moving target. Their task was to halt the moving target (the experimenter) at exactly $140 \mathrm{~cm}$ by asking the experimenter to stop when the experimenter reached that distance. After the subjects understood the task, they were taken to a new location, where they encountered either a male or female experimenter who had been prepositioned to stand at a specific location on a $210-\mathrm{cm}$ line marked on the plaza cement. The subjects were instructed to stand with their toes touching one end of the line. The target/experimenter stood facing the subject and the target/ experimenter's initial positioning along the line created the approaching or departing condition.

In the approaching condition, the experimenter was prepositioned $210 \mathrm{~cm}$ from the subject at the opposite end of the line. In the departing condition, the experimenter was prepositioned to stand $70 \mathrm{~cm}$ from the subject's end of the line. After the subject was properly positioned, the experimenter moved slowly (approximately $2 \mathrm{sec}$ per step) from the initial starting point toward the $140-\mathrm{cm}$ distance. Thus, if the experimenter started at $210 \mathrm{~cm}$, this meant approaching the subject as the experimenter moved toward $140 \mathrm{~cm}$; if the experimenter started from $70 \mathrm{~cm}$, this meant moving away from the subject as the experimenter moved toward $140 \mathrm{~cm}$.

In either case, the subject's task was to stop the moving experimenter when the experimenter reached what the subject thought was $140 \mathrm{~cm}$. There were no distance markings on the line, and after the subject halted the experimenter by saying "stop," the toe-to-toe distance between the 
Table 1

Means and Standard Deviations (SD) of the Measured Distances (in Centimeters)

\begin{tabular}{|c|c|c|c|c|c|c|c|c|c|c|}
\hline \multirow[b]{3}{*}{ Condition } & \multicolumn{4}{|c|}{ Female Subject } & \multicolumn{4}{|c|}{ Male Subject } & \multirow{2}{*}{\multicolumn{2}{|c|}{ Combined }} \\
\hline & \multicolumn{2}{|c|}{ Male Exp. } & \multicolumn{2}{|c|}{ Female Exp. } & \multicolumn{2}{|c|}{ Male Exp. } & \multicolumn{2}{|c|}{ Female Exp. } & & \\
\hline & $M$ & $S D$ & $M$ & $S D$ & $M$ & $S D$ & $M$ & $S D$ & $M$ & $S D$ \\
\hline Approaching from $210-\mathrm{cm}$ start & 177.12 & 29.83 & 187.12 & 48.30 & 172.11 & 33.47 & 174.42 & 56.29 & 177.62 & 40.18 \\
\hline $\begin{array}{l}\text { Departing from } 70-\mathrm{cm} \text { start } \\
\text { Combined }\end{array}$ & $\begin{array}{l}151.75 \\
164.43\end{array}$ & 22.97 & $\begin{array}{l}167.50 \\
177.31\end{array}$ & 35.35 & $\begin{array}{l}156.66 \\
164.38\end{array}$ & 34.21 & $\begin{array}{l}156.00 \\
165.21\end{array}$ & 55.77 & 158.00 & 36.04 \\
\hline
\end{tabular}

Note $-n=8$ in each cell. Exp. = experimenter.

subject and the experimenter was measured with a tape measure. Toeto-toe and nose-to-nose distances are very similar, so these distances were considered to be what the subject perceived as $140 \mathrm{~cm}$ from the human target.

The 70-140-, and 210-cm distances were chosen in order to provide the same required time and $70-\mathrm{cm}$ moving distance for the experimenter to reach the $140-\mathrm{cm}$ target distance. The $70-\mathrm{cm}$ closest subjectexperimenter distance approximates the average distance of the onset of "slight discomfort," as reported in Hayduk (1981). The considerable individual variations in personal-space preferences imply that the 70 -cm starting distance was somewhere between "almost no encroachment" to, at most, a "moderate encroachment" on the subject's spatial preference. Thus, in the departing condition, the experimenter was departing from a condition of very mild to moderate spatial encroachment, whereas in the approaching condition, the experimenter should never have approached close enough to trigger a report of even slight discomfort from the subject.

\section{RESULTS}

The means and standard deviations of the measured distances are presented in Table 1.

Analyzing these data with a three-way analysis of variance (subject's sex $\times$ experimenter's sex $\times$ approachingdeparting) located no interactions and only one significant main effect. The subjects halted the experimenter at significantly larger distances in the approaching than in the departing condition $[F(1,56)=28.01, p<.01]$, irrespective of the sex of the subject and the experimenter.

We will not speculate about the reasons for the lack of sex effects but will note that this merely adds a tad of evidence to the more than 70 studies cited in Hayduk (1983, p. 309). The absence of sex effects implies that the effects produced by approaching or departing hold for all pairings of the sex of the subject and the experimenter.

Figure 1 provides a visual summary of the significant differences between the approaching/departing means. In both the departing and the approaching conditions, the subjects tended to underestimate the target/experimenter's distance. The real distance remaining between the subject and the experimenter (on average, 158 or $178 \mathrm{~cm}$ ) was larger than the 140-cm estimate the subject attested to by saying "stop." Since their $140-\mathrm{cm}$ estimate was typically smaller than the actual distance, the subjects were implicitly reporting that the experimenter "felt" closer to them than the experimenter actually was.

Furthermore, those whose personal spaces were never encroached on (those in the approaching condition) displayed more underestimation than those whose spaces had been mildly encroached on (those in the departing condition). The average distance for the approaching condition

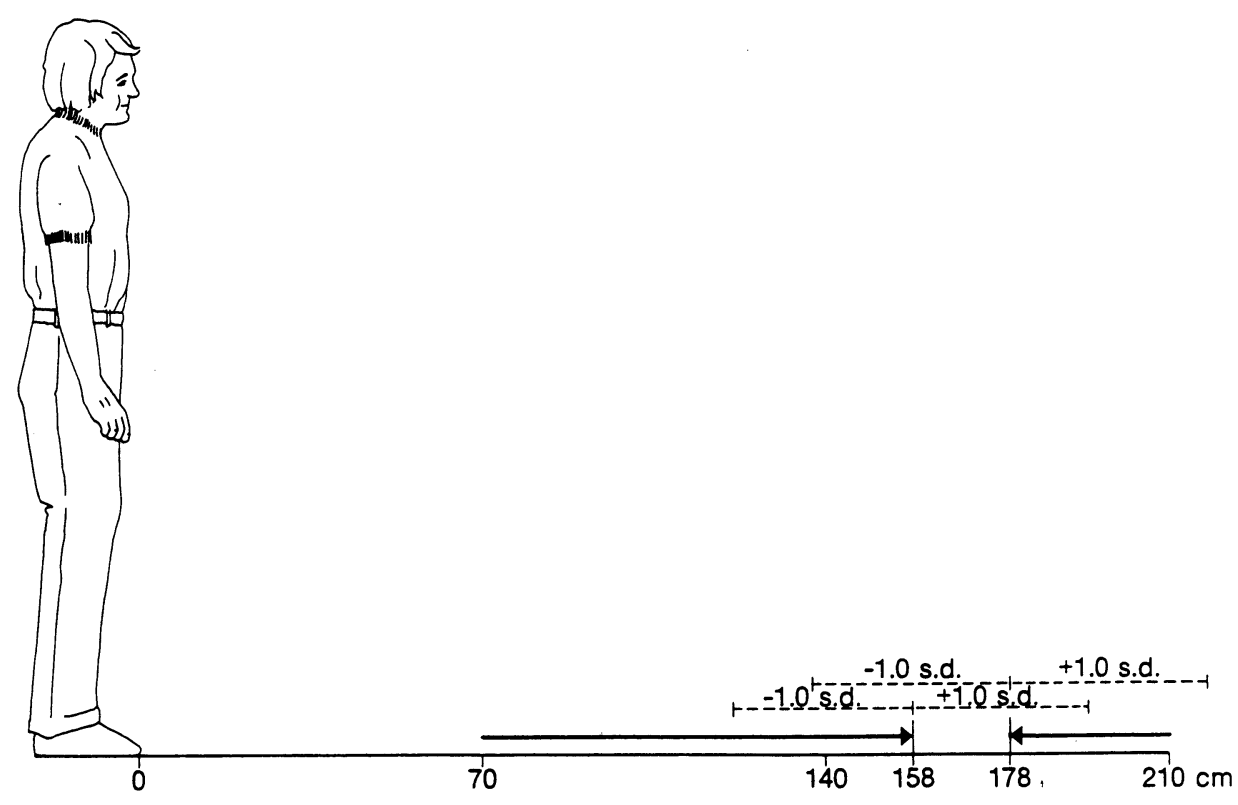

Figure 1. A schematic representation of the results of the approaching and departing conditions. 
is about twice as far from the true $140-\mathrm{cm}$ distance as is the average for the departing condition.

It would be incorrect to conclude that the average size of underestimation for the approaching group is double that for the departing group. The substantial standard deviations represented in Figure 1 imply that the "average" sizes of the estimation errors (the average of the absolute difference between $140 \mathrm{~cm}$ and each subject's response) is considerably larger than the differences between $140 \mathrm{~cm}$ and the means for each of the groups. That is, the difference between the group means is relatively modest compared with the individual variations in stopping distances within the groups.

\section{DISCUSSION}

Are these observations consistent with "perceptual withdrawal," if by "perception" we mean awareness of an environment through reception of physical cues, and by "withdrawal" we mean acting in a way that limits the input to a system in order to reduce the load on some system component? We think not. The people subjected to the greatest load, and hence those most motivated toward withdrawal through misperception, displayed less misperception, on average, than those with no seeming motivation. The mild spatial intrusion implied by the 70 cm starting distance resulted in less, not more, misperception.

One might argue that the arousal caused by a slight intrusion alerted the subjects sufficiently so that they paid more attention and thus made smaller errors. But then one would have to explain why the standard deviations in the two groups differ only minimally and why one should observe an implicit baseline of "substantial errors" in the first place.

Nor do current personal-space theories assist us (see Hayduk, 1983, pp. 303-307, for a summary). The current theories focus on intrusions and the inner boundary of personal space and are simply ill-suited to account for phenomena at the outer limits of personal space, at which there is the least arousal to label or attribute, little to reequilibrate, and no substantial violation of expectations.

In the most comparable study we were able to locate, Ford, Knight, and Cramer (1977) report that when subjects maintained an intrusive $30.5-\mathrm{cm}$ distance from a confederate for $5 \mathrm{sec}$, the distance was perceived as only $18.56 \mathrm{~cm}-$ so their subjects also underestimated the distance to an intruder. They explained their observations by employing Werner and Wapner's (1955) sensory-tonic field theory, which links one's experiences to a biological base for maintaining equilibrium. Essentially, this theory predicts that if subjects are required to counteract some primary force on the body to maintain equilibrium, perception will change. In particular, "if the counteractive force is forward, the object appears closer" (p. 165). If we view the increasing resistance to closer personal spacings as a primary force producing a backward lean that is counteracted by a compensating forward-lean force, Werner and Wapner would correctly predict that the target/experimenters would appear closer than they actually were. But Werner and Wapner would have difficulty with our observation that those subjects receiving the stronger forces (those in the departing condition) displayed less underestimation. It might be possible to salvage this theory by incorporating "relative rates of force change" into the theory, but this requires some fundamental theoretical revisions.

The notion of an "impending," "anticipating," or a "forward-looking system" seems crucial to accounting for a stronger effect in the approaching condition. Anticipatory systems must have an internal prediction for the future and hence must have two senses of time: clock time for perceiving the current action (the approaching experimenter) and time as a constituent feature of the internal predictive device for the developing matter that is thought about (cf. Garfinkel's [1967, p. 40] distinction between clock time and time as a constituent feature of "the matter being talked about"). Any discussion of the temporal aspects of internal mental calculations, however, takes us toward the cognitive and away from our initial, possibly naive, view that this could be a "merely perceptual" phenomenon.

We are forced to ponder anew the phenomenology and gestalt perceptual characteristics of the experience of someone approaching or moving away from oneself and to focus more specifically on the anticipatory, predictive, and constructive nature of human spatial experiences.

Could misestimation of distance be used as a measure of personal space? The key to deciding this is to determine the aspect of personal space that is represented by erroneous judgments. The substantial withingroup standard deviations inform us that there are indeed considerable individual differences in estimation abilities, but the data from this current study provide no evidence of the characteristics of the individuals displaying the greatest misestimation. Our approaching/departing data were not created by considering individual characteristics but by creating differing conditions to which the individuals were subjected. Clearly, individual differences in distance misestimation for nonhuman objects and the "standard" sources of misperception-liking, familiarity, status, and affective relationships (cf. Tajfel, 1969)-should all be considered.

In particular, it would be important to determine whether distance misestimation is linked to personal space as measured either by the stop distance or by other procedures. If this link were weak, we would have some assurance that we are measuring some separate aspect of human spatial behavior. A strong correlation, however, could make the standard personal-space measurements mere artifacts of the more fundamental cognitive mechanisms producing misestimation or miscognition.

\section{REFERENCES}

Ford, J. G., KNIGHT, M., CRAmer, R، (1977). The phenomenological experience of interpersonal spacing. Sociometry, 40, 387-396.

GARFINKEL, H. (1967). Studies in ethnomethodology. Englewood Cliffs: Prentice-Hall.

HAyduK, L. A. (1978). Personal space: An evaluative and orienting overview. Psychological Bulletin, 85, 117-134.

HAYDUK, L. A. (1981). The permeability of personal space. Canadian Journal of Behavioural Science, 13, 274-287.

Hayduk, L. A. (1983). Personal space: Where we now stand. Psychological Bulletin, 94, 293-335.

McNamara, T. P., Ratcliff, R., \&cKoon, G. (1984). The mental representation of knowledge acquired from maps. Journal of Experimental Psychology: Leaming, Memory, \& Cognition, 10, 723-732.

RUSSELl, J. A., \& WARD, L. M. (1982). Environmental psychology. Annual Review of Psychology, 33, 651-688.

Stevens, A., \& Coupe, P. (1978). Distortions in judged spatial relations. Cognitive Psychology, 10, 422-437.

TAJFEL, H. (1969). Social and cultural factors in perception. In G. Lindsay \& E. Aronson (Eds.), Handbook of social psychology (2nd ed., pp. 315-394). Reading, MA: Addison-Wesley.

TERRY, R. L., \& LowER, M. (1979). Perceptual withdrawal from an invasion of personal space: A methodological note. Personality \& Social Psychology Bulletin, 5, 396-397.

WERNER, H., \& WAPNER, S. (1955). Changes in psychological distance under conditions of danger. Journal of Personality, 24, 153-167.

(Manuscript received July 5, 1991.) 